\title{
Alteration of Prolactin, Thyroid Hormones and Lipid Profiles during Three Stages of Pregnancy
}

\author{
Khalid Shaalan Sahab \\ Department of Chemistry, College of Science, Diyala University, Diyala, Iraq \\ e-mail: khalidshaalan1970@yahoo.com
}

\section{Abstract}

Pregnancy causes a change in both hormonal and biochemical content of the maternal blood. Therefore, this study was to investigate the changing in TSH, T3, T4, prolactin and lipid profile during the three stage of pregnancy. The study included 30 pregnant women and 20 non-pregnant women as control group. Hormones were measured by hormones automated analyzer (Cobase400 Integra Plus, Roche, Germany) and lipid profiles were measured by biochemistry automated analyzer (Cobase411, Roche, Germany). The results showed significant elevation in, prolactin, in three stage of pregnancy $(p<0.05)$. While T3, T4, TC and TG were showed no significant elevation $(p>0.05)$ in first trimester when compared to control group, but TC and TG increased significantly $(p<0.05)$ in the second stage and third stage of pregnancy trimester and T3 and T4 elevated significantly $(p<0.05)$ in third stage of trimester. LDL and HDL were showed no significant elevation $(p>0.05)$ in all stages of trimester when compared to control group.

Conclusion: The normal period of pregnancy is accompanied with elevation of prolactin, thyroid hormones and increase of lipids fractions.

\section{Introduction}

The period of reproduction in human is known as gestation, gravidity, as well as pregnancy. In this period of reproduction or "pregnancy" a woman carries one or more live offspring from implantation of a fertilized zygote in the uterus. So, pregnancy is the time during which one or more offspring develops inside a woman's womb. Pregnancy Received: February 3, 2019; Accepted: February 11, 2019

Keywords and phrases: thyroid hormones, prolactin, lipids, three stages of pregnancy.

Copyright (C) 2019 Khalid Shaalan Sahab. This is an open access article distributed under the Creative Commons Attribution License, which permits unrestricted use, distribution, and reproduction in any medium, provided the original work is properly cited. 
has a great impact on the internal and external physiological status of woman. During this pregnancy period there are several internal physiological changes occur for pregnant woman. The internal physiological changes happened for pregnant woman involves alteration in blood parameters and these changes may appear to be pathological in the non-pregnant state $[1,2]$. These changes related to the growth and development the fetus [2]. Therefore, in pregnancy, woman physiology is altered greatly to supply the developing fetus with suitable nutrients required for growth and provide the protective to fetus until delivery, and also, to accommodate the mother with additional energy required for labour. The normal woman pregnancy period lasts for 40 weeks (about 280 days), divided into three stages "trimesters" according to the period of growth and development the fetus [1-4].

1. First trimester (up to 13 weeks after conception) where the fetus weighs approximately $13 \mathrm{~g}$ and is up to $8 \mathrm{~cm}$ long.

2. Second trimester (13 to 26 weeks), fast growth occurs for fetus and in the end of this trimester, the fetus weighs approximately $70 \mathrm{~g}$ and is $30 \mathrm{~cm}$ long within which the fetal organs would have begun to mature.

3. Third trimester (26-40 weeks), the fetal organs complete maturation.

Pregnancy is characterized by changes in a woman's metabolism and hormonal status during these three stages. Therefore a study like these variations is necessary to assess the influence of normal pregnancy on blood profiles [3-5].

Thyroid gland is critical in trimesters as it regulates the production of two hormones each of which play an important role in the development of the nervous system and brain of baby, these thyroid hormones are triiodothyronine (T3) and thyroxine (T4). In first trimester, the fetus depends on thyroid hormones of his mother supplied to him through the placenta. This additional need of thyroid hormones leads to enlargement of the thyroid gland of mother, to increase the thyroid hormones production. Adequate circulating levels of thyroid hormones are necessary for normal reproductive function. Disorders of thyroid glands are common in women during period of pregnancy. If untreated, both hyper- and hypo-thyroidism are conjugated with reverse effects on pregnancy and fetal outcomes. The correct identifying of these disorders and treat them it is necessary to prevent pregnancy-related complications [6]. The production of T3 and T4 is triggered by induce of thyroid stimulating hormone (TSH) that produced and secreted from pituitary gland. 
Physiologically, the mother becomes a new person during the period of pregnancy, and maternal metabolic environment is modified by increase in blood levels of progesterone, estrogens and, hyperplasia of pancreatic beta-cells. Insulin resistance and ovaries-estrogens (in women) play an important role in disturbing lipid profile. The association of pregnancy with high concentrations of o-estrogens and some form of insulin resistance may contribute to disturbed lipid profile especially in the third trimester of pregnancy [7]. In this study we investigate the changes in lipids profile, T3, $\mathrm{T} 4, \mathrm{TSH}$, and prolactin in a group of pregnant women to determine the changing these parameters during normal pregnancy.

\section{Materials and Methods}

This study was carried out in AL-Batol teaching Hospital of Baquba City, Diyala Governorate, Iraq for a period of October to May, 2017. A total of 50 women between the ages of 20 and 41 years volunteered for the study. This 50 volunteer women included 30 pregnant women and 20 non-pregnant women as control group. The 30 pregnant women were studied for about 32th week of pregnancy according to stage of pregnancy.

Samples of blood were taken from all participate women after made them fast overnight for 8-12 hrs. $5 \mathrm{ml}$ of fasting venous blood was collected from each woman using a $5 \mathrm{ml}$ disposable syringes and transferred into plain tube. The blood was left to be clotted for half hour then centrifuged at $4000 \mathrm{rpm}$ for 5 minutes and used to assay the studied parameters. Serum total cholesterol (TC), triglycerides (TG) and HDL cholesterol (HDL-C) were analyzed by using biochemistry automated analyzer (Cobase411, Roche, Germany). Hormones were measured by hormones automated analyzer (Cobase400Integra Plus, Roche, Germany). Estimation of low density lipoprotein- cholesterol (LDL-C) was done by using the Friedewald formula.

Data analysis performed with using the SPSS statistical software and results were presented as mean \pm standard deviation $(\mathrm{M} \pm \mathrm{SD})$. The student $t$-test was used for analyses of data and $p<0.05$ were considered statistically significant.

\section{Results}

The mean \pm standard division $(\mathrm{M} \pm \mathrm{SD})$ values of studied blood parameters for the control and pregnant women in three stages of trimester are shown in Table 1. 
Table 1. $M \pm S D$ values of studied parameters in control and the three trimesters of pregnancy.

\begin{tabular}{|c|c|c|c|c|}
\hline Parameters & Control & 1st trimester & 2nd trimester & 3rd trimester \\
\hline TSH mIU/L & $3.50 \pm 1.08$ & $2.90 \pm 1.23$ & $3.40 \pm 0.69$ & $3.50 \pm 1.15$ \\
\hline TT3 nmol/L & $2.00 \pm 0.30$ & $3.20 \pm 0.44$ & $2.90 \pm 0.96$ & $2.60 \pm 0.47$ \\
\hline TT4 nmol/L & $90.00 \pm 12.58$ & $138.0 \pm 22.05$ & $140.0 \pm 34.22$ & $150.0 \pm 62.31$ \\
\hline Prolactin $\mu \mathrm{g} / \mathrm{L}$ & $15.00 \pm 3.01$ & $34.00 \pm 32.75$ & $100.0 \pm 33.45$ & $131.1 \pm 31.44$ \\
\hline $\mathrm{TC} \mathrm{mg} / \mathrm{dl}$ & $150.4 \pm 17.4$ & $166.4 \pm 28.1$ & $180.0 \pm 23.4$ & $207.3 \pm 32.2$ \\
\hline $\mathrm{TG} \mathrm{mg} / \mathrm{dl}$ & $80.7 \pm 6.6$ & $108.7 \pm 13.1$ & $126.5 \pm 18.4$ & $130.7 \pm 20.8$ \\
\hline HDL-C mg/dl & $43.1 \pm 5.4$ & $44.7 \pm 4.2$ & $48.0 \pm 6.3$ & $52.0 \pm 5.8$ \\
\hline LDL-C mg/dl & $81.8 \pm 14.6$ & $90.3 \pm 9.6$ & $123.7 \pm 10.9$ & $159.5 \pm 22.4$ \\
\hline
\end{tabular}

As shown in Table 2 the results for comparison of studied parameters in first trimester with control group have been showed no significant differences between blood levels of these parameters except in prolactin which showed significant elevation in first trimester as comparison to control $(p<0.05)$.

Table 2. Showed the comparison of studied parameters in first trimester with control.

\begin{tabular}{|c|c|c|c|}
\hline Parameters & Control & 1st trimester & P-value \\
\hline TSH & $3.50 \pm 1.08$ & $2.90 \pm 1.23$ & 0.784 \\
\hline T3 & $2.00 \pm 0.30$ & $3.20 \pm 0.44$ & 0.234 \\
\hline T4 & $90.00 \pm 12.58$ & $138.0 \pm 22.05$ & 0.439 \\
\hline Prolactin & $15.00 \pm 3.01$ & $34.00 \pm 32.75$ & $0.01 *$ \\
\hline TC & $150.4 \pm 17.4$ & $166.4 \pm 28.1$ & 0.195 \\
\hline TG & $80.7 \pm 6.6$ & $108.7 \pm 13.1$ & 0.125 \\
\hline HDL-C & $43.1 \pm 5.4$ & $44.7 \pm 4.2$ & 0.254 \\
\hline LDL-C & $81.8 \pm 14.6$ & $90.3 \pm 9.6$ & 0.321 \\
\hline
\end{tabular}

The result in Table 3 showed there are elevation in levels of studied parameters but without significant differences $(p>0.05)$, except in levels of prolactin, TC and TG which showed presence significant elevation $(p<0.05)$ in second trimester in comparison to control group and TSH showed nearly normalization. 
Table 3. Showed the comparison of studied parameters in second trimester with control.

\begin{tabular}{|c|c|c|c|}
\hline Parameters & Control & 2nd trimester & P-value \\
\hline TSH & $3.50 \pm 1.08$ & $3.40 \pm 0.69$ & 0.72 \\
\hline T3 & $2.00 \pm 0.30$ & $2.90 \pm 0.96$ & 0.089 \\
\hline T4 & $90.00 \pm 12.58$ & $140.0 \pm 34.22$ & 0.077 \\
\hline Prolactin & $15.00 \pm 3.01$ & $100.0 \pm 33.45$ & $0.000^{*}$ \\
\hline TC & $150.4 \pm 17.4$ & $180.0 \pm 23.4$ & $0.049^{*}$ \\
\hline TG & $80.7 \pm 6.6$ & $126.5 \pm 18.4$ & $0.045^{*}$ \\
\hline HDL-C & $43.1 \pm 5.4$ & $48.0 \pm 6.3$ & 0.231 \\
\hline LDL-C & $81.8 \pm 14.6$ & $123.7 \pm 10.9$ & 0.063 \\
\hline
\end{tabular}

The result showed there are significant elevation $(p<0.05)$, in levels of most studied parameters except in levels of HDL and LDL which showed presence elevation without significant differences $(p>0.05)$ in second trimester in comparison to control group and TSH showed normalization as shown in Table 4.

Table 4. Showed the comparison of studied parameters in third trimester with control.

\begin{tabular}{|c|c|c|c|}
\hline Parameters & Control & 3rd trimester & P-value \\
\hline TSH & $3.50 \pm 1.08$ & $3.50 \pm 1.15$ & 0.733 \\
\hline T3 & $2.00 \pm 0.30$ & $2.60 \pm 0.47$ & $0.023^{*}$ \\
\hline T4 & $90.00 \pm 12.58$ & $150.0 \pm 62.31$ & $0.017^{*}$ \\
\hline Prolactin & $15.00 \pm 3.01$ & $131.1 \pm 31.44$ & $0.000^{*}$ \\
\hline TC & $150.4 \pm 17.4$ & $207.3 \pm 32.2$ & $0.042^{*}$ \\
\hline TG & $80.7 \pm 6.6$ & $130.7 \pm 20.8$ & $0.044^{*}$ \\
\hline HDL-C & $43.1 \pm 5.4$ & $52.0 \pm 5.8$ & 0.09 \\
\hline LDL-C & $81.8 \pm 14.6$ & $159.5 \pm 22.4$ & 0.051 \\
\hline
\end{tabular}

\section{Discussion}

The results showed increase the levels of total thyroid hormones TT3 and TT4 in pregnant women especially in third stage of trimester. This result agreed with the scientific fact that says the thyroid gland, during pregnancy, is hyper-stimulated and resulting increases in thyroid hormone concentrations to increases the basal metabolism 
rate adequate for mother and fetus. So, during pregnancy accurate assessment of thyroid function is critical for the adjustment of thyroid hormone. During pregnancy when thyroid is insufficient this case may associate with adverse maternal obstetric outcome and lead to child neuro developmental deficits. At present time there are a gestational age-specific reference intervals available for tests of thyroid function which are critical for maintaining the delicate balance of thyroid hormones during pregnancy. Knowing the expected normal changes in thyroid hormone concentrations during pregnancy allows, when necessary, individualized supplementation [8]. Women who have been diagnosed with thyroid gland dysfunctions are usually treated and are able to complete a normal pregnancy [8-10]. The period of pregnancy induces complex changes in thyroid binding globulin (TBG) concentration and in circulating maternal steroid hormones (estrogens). Estrogens have stimulatory effects on TBG synthesis, and total T4 and total T3 concentration increase in increases in serum TBG concentrations. The concentrations of TBG double by weeks 16 to 20 of gestation [11, 12]. Thyroid-stimulating hormone (TSH) levels are low in the first trimester, with normalization by the second and third trimester. Pregnancy can be a stress for the thyroid and normally the size of the gland increases by $10 \%$. Therefore, the production of T3 and T4 increases by about $50 \%$. As a result, the normal TSH level during pregnancy is lower than or normal as compared to non-pregnancy level [8, 13]. Because normal thyroid function is different during pregnancy, TSH values will change as the mother progresses from the first to third trimester. Under normal circumstances, the normal TSH value would range from 0.2 to $4.0 \mathrm{mlU} / \mathrm{L}$.

Prolactin concentration in this study revealed significant elevation in the first trimester when compared to control group and also increased progressively with high significant elevation in second and third stages of pregnancy. This elevation is to preparation and induce the mammalian gland for secretion of milk for feeding the neonate. This result agreement with some previous studies [5, 14-17].

The current study revealed increases of lipid fractions (TC, TG, HDL and LDL). The growth and development of the fetus and support its structures during pregnancy period demand increases the metabolic fuels. Lipids are high energy food. During pregnancy lipids are essential classes of food that provide energy for the multisystem of the mother and for increased cellular proliferation of the fetus. This is necessary because of the high energy required for the increased cellular proliferation of maternal uterine enlargement, blood volume expansion, fetal implantation, formation of blood vessels in the utero 
placenta area, feto-placenta development and growth [21-24]. Throughout normal pregnancy advances high concentrations of many of steroids are required. Since most steroids synthesized from cholesterol, its amounts increased in the circulation of normal pregnant women. Various previous studies observed an increase in lipid fractions and thought that the increase in lipids of blood is related to development of the mammary apparatus and to the requirements of the fetus [1, 18-20]. Thus high lipid profile is a normal finding. Nevertheless, a hyperlipidemia increases the risk of coronary artery disease $(\mathrm{CAD})$ and can adversely affect the health of the pregnant woman and her fetus [25].

\section{Conclusion}

The normal period of pregnancy is accompanied with elevation of prolactin, thyroid hormones and increase of lipids fractions. If there were low levels of thyroid hormones that mean the pregnant is suffering from hypothyroidism and must be treated. High prolactin is normal to enhance the mammalian gland for secretion of milk for lactating the neonate. High lipid fractions are to provide the fetus with energy and compounds that necessary to builds its structures. Also, lipids are essential classes of food that provide multisystem of the mother with energy.

\section{References}

[1] Azab Elsayed Azab, Mohamed Omar Albasha and Sara Yosef Elhemady, Haematological parameters in pregnant women attended antenatal care at Sabratha Teaching Hospital in Northwest, Libya, Pathology and Laboratory Medicine 1(2) (2017), 61-69.

[2] Alkhansa Osama Mohamed, Khalda Mirghani Hamza and Asaad Mohammed Ahmed Babker, Physiological changes in some hematological and coagulation profile among Sudanese healthy pregnant women, Int. J. Med. Sci. Public Health 5(3) (2016), 525-528.

[3] Olusegun Taiwo Oke, Stephen Olajide Awofadeju and Samuel Oyewole Oyedeji, Haemorheological profiles in different trimesters among pregnant women in South West Nigeria, Pak. J. Physiol. 7(2) (2011), 17-19.

[4] Marilynn C. Frederiksen, Physiologic changes in pregnancy and their effect on drug disposition, Seminars in Perinatology 25(3) (2001), 120-123.

[5] O. Akinloye, O. M. Obikoya, A. I. Jegede, D. P. Oparinde and A. O. Arowojolu, Cortisol plays central role in biochemical changes during pregnancy, Int. J. Med. Biomed. Res. 2(1) (2013), 3-12. 
[6] Pratap Kumar and Navneet Magon, Hormones in pregnancy, Niger Med. J. 53(4) (2012), 179-183.

[7] Sinan Butrus Garabet, Lipid profile in normal pregnancy, Zanco J. Med. Sci. 16(3) (2012), 194-198.

[8] Offie P. Soldin, Thyroid function testing in pregnancy and thyroid disease: trimesterspecific reference intervals, Ther. Drug Monit. 28(1) (2006), 8-11.

[9] R. Z. Klein, J. E. Haddow, J. D. Faix et al., Prevalence of thyroid deficiency in pregnant women, Clin. Endocrinol. (Oxf.) 35 (1991), 41-46.

[10] B. M. Casey, J. S. Dashe, C. E. Wells et al., Subclinical hypothyroidism and pregnancy outcomes, Obstet. Gynecol. 105 (2005), 239-245.

[11] D. Glinoer, What happens to the normal thyroid during pregnancy?, Thyroid 9 (1999), 631-635.

[12] L. Bartalena, Recent achievements in studies on thyroid hormone-binding proteins, Endocr Rev. 11 (1990), 47-64.

[13] Nosratollah Zarghami, Mohammad Rohbani-Noubar and Ali Khosrowbeygi, Thyroid hormones status during pregnancy in normal Iranian women, Indian Journal of Clinical Biochemistry 20(2) (2005), 182-185.

[14] M McLean and R. Smith, Cushing's syndrome: how should we investigate in 1995?, The Med. J. Austral. 1637 (1995), 153-154.

[15] L. G. Sobrinho, Prolactin, psychological stress and environment in humans: adaptation and maladaptation, Pituitary 6 (2003), 35-39.

[16] A. Kratz, M. Ferraro, P. M. Sluss and K. B. Lewandrowski, Normal reference laboratory values, N. Engl. J. Med. 351(15) (2004), 1548-1563.

[17] M. Abbassi-Ghanavati, L. G. Greer and F. G. Cunningham, Pregnancy and laboratory studies: a reference table for clinicians, Obstet. Gynecol. 114(6) (2009), 1326-1331.

[18] A. A. Akinbami, S. O. Ajibola, K. A. Rabiu, A. A. Adewunmi, A. O. Dosunmu, A. Adediran, V. O. Osunkalu, B. I. Osikomaiya and K. A. Ismail, Hematological profile of normal pregnant women in Lagos, Nigeria, Inter. J. Women Health 5 ( 2013), 227-232.

[19] G. Stevens, M. Finucane, L. De-Regil, C. Paciorek, S. Flaxman, F. Branca et al., Nutrition impact model study group (Anaemia). Global, regional, and national trends in haemoglobin concentration and prevalence of total and severe anaemia in children and pregnant and non-pregnant women for 1995-2011: A systematic analysis of populationrepresentative data, Lancet Glob. Health 1 (2013), 16-25. 
[20] D. B. Jiji and K. A. Rajagopal, A study to assess the knowledge and risk factors of anaemia among the pregnant women attending selected health care facilities in Sebha, Libya, J. Sci. 4(1) (2014), 19-22.

[21] F. Boehlen, P. Hohlfeld, P. Extermann, T. V. Perneger and P. De Moerloose, Platelet count at term pregnancy: A reappraisal of the threshold, Obstet. Gynecol. 95(1) (2000), 29-33.

[22] J. D. Jensen, S. E. Wiedmeier, E. Henry, R. M. Silver and R. D. Christensen, Linking maternal platelet counts with neonatal platelet counts and outcomes using the data repositories of a multihospital health care system, Am. J. Perinatol. 28(8) (2011), 597604.

[23] K. E. Onwukeme and V. E. Uguru, Haematological values in pregnancy in Jos, Afr. J. Med. 9(2) (1990), 70-75.

[24] Deepak Parchwani and Digisha Patel, Status of lipid profile in pregnancy, National Journal of Medical Research 1(1) (2011), 10-12.

[25] R. A. Elzahaf and M. Omar, Prevalence of anaemia among pregnant women in Derna city, Libya, Int. J. Community Med. Public Health 3(7) (2016), 1915-1920. 